\title{
Cell-Surface and Secreted Isoforms of CSF-1 Exert Opposing Roles in Macrophage-Mediated Neural Damage in Cx32- Deficient Mice
}

\author{
Janos Groh, ${ }^{1}$ Ranu Basu, ${ }^{2}$ E. Richard Stanley, ${ }^{2}$ and Rudolf Martini ${ }^{1}$ \\ ${ }^{1}$ Department of Neurology, Developmental Neurobiology, University Hospital Wuerzburg, D-97080 Wuerzburg, Germany, and 2Department of \\ Developmental and Molecular Biology, Albert Einstein College of Medicine, Bronx, New York 10461
}

Previous studies in myelin-mutant mouse models of the inherited and incurable nerve disorder, Charcot-Marie-Tooth (CMT) neuropathy, have demonstrated that low-grade secondary inflammation implicating phagocytosing macrophages amplifies demyelination, Schwann cell dedifferentiation and perturbation of axons. The cytokine colony stimulating factor-1 (CSF-1) acts as an important regulator of these macrophage-related disease mechanisms, as genetic and pharmacologic approaches to block the CSF-1/CSF-1R signaling result in a significant alleviation of pathological alterations in mutant peripheral nerves. In mouse models of CMT1A and CMT1X, as well as in human biopsies, CSF-1 is predominantly expressed by endoneurial fibroblasts, which are closely associated with macrophages, suggesting local stimulatory mechanisms. Here we investigated the impact of cell-surface and secreted isoforms of CSF- 1 on macrophagerelated disease in connexin32-deficient (Cx32def) mice, a mouse model of CMT1X. Our present observations suggest that the secreted proteoglycan isoform (spCSF-1) is predominantly expressed by fibroblasts, whereas the membrane-spanning cell-surface isoform (csCSF-1) is expressed by macrophages. Using crossbreeding approaches to selectively restore or overexpress distinct isoforms in CSF-1-deficient (osteopetrotic) Cx32def mice, we demonstrate that both isoforms equally regulate macrophage numbers dosedependently. However, spCSF-1 mediates macrophage activation and macrophage-related neural damage, whereas csCSF-1 inhibits macrophage activation and attenuates neuropathy. These results further corroborate the important role of secondary inflammation in mouse models of CMT1 and might identify specific targets for therapeutic approaches to modulate innate immune reactions.

Key words: CMT1X; colony stimulating factor-1; demyelination; macrophage

Significance Statement

Mouse models of Charcot-Marie-Tooth neuropathy have indicated that low-grade secondary inflammation involving phagocytosing macrophages amplifies demyelination, Schwann cell dedifferentiation, and perturbation of axons. The recruitment and pathogenic activation of detrimental macrophages is regulated by CSF-1, a cytokine that is mostly expressed by fibroblasts in the diseased nerve and exists in three isoforms. We show that the cell-surface and secreted isoforms of CSF-1 have opposing effects on macrophage activation and disease progression in a mouse model of CMT1X. These insights into opposing functions of diseasemodulating cytokine isoforms might enable the development of specific therapeutic approaches.

\section{Introduction}

Charcot-Marie-Tooth (CMT) diseases are the most common type of inherited peripheral neuropathies and can severely impair the quality-of-life via motor and sensory malfunctions (Patzko

\footnotetext{
Received Sept. 14, 2015; revised Dec. 1, 2015; accepted Dec. 24, 2015.

Author contributions: J.G., E.R.S., and R.M. designed research; J.G. and R.B. performed research; R.B. and E.R.S. contributed unpublished reagents/analytic tools; J.G. and R.M. analyzed data; J.G., E.R.S., and R.M. wrote the paper.

This work was supported by the German Research Foundation (MA 1053/6-1 to R.M.), the Interdisciplinary Centre for Clinical Research (IZKF) of the University of Würzburg (A-122 to R.M.) and National Institutes of Health Grant CA32551 (E.R.S.). We thank Heinrich Blazyca, Silke Loserth, and Bettina Meyer for expert technical assistance, and Helga Brünner, Jacqueline Schreiber, Anja Weidner, and Jennifer Bauer for attentive care of mice.

The authors declare no competing financial interests.
}

and Shy, 2011; Johnson et al., 2014; Pareyson et al., 2014). Despite significant progress in research regarding disease-causing mutations and pathomechanisms, there is presently no effective therapy available (Jerath and Shy, 2015).

Numerous rodent models of different CMT subtypes are available and studies in these models revealed differences but also highlighted common aspects between the distinct forms (Fle-

Correspondence should be addressed to either Dr. Janos Groh or Rudolf Martini, Department of Neurology, Developmental Neurobiology, University Hospital Wuerzburg, Josef-Schneider-Str. 11, D-97080 Wuerzburg, Germany, E-mail: groh_j@ukw.de or rudolf.martini@mail.uni-wuerzburg.de.

DOI:10.1523/JNEUROSCI.3427-15.2016

Copyright $\odot 2016$ the authors $\quad 0270-6474 / 16 / 361890-12 \$ 15.00 / 0$ 
drich et al., 2012; Groh et al., 2015a). An important feature common to several models is the occurrence of low-grade secondary inflammation in the diseased peripheral nerves, with cells of the adaptive and innate immune system functioning as amplifiers of the demyelinating and axonopathic disease (Groh et al., 2015a).

A particularly potent disease mechanism is mediated by colony stimulating factor-1 (CSF-1), the primary regulator of the development and maintenance of tissue macrophages (Cecchini et al., 1994). In nerves of CMT1 mouse models, as well as in nerve biopsies from CMT1A and CMT1X patients, CSF-1 is predominantly expressed by endoneurial fibroblasts (Groh et al., 2012), a connective tissue-related cell type with multiple functions, but still under-researched roles in nerve disorders (Richard et al., 2012). When CSF-1 was inactivated by cross-breeding with osteopetrotic $\left(C s f 1^{o p / o p}\right)$ mice, a spontaneous null mutant for CSF-1 (Wiktor-Jedrzejczak et al., 1990; Yoshida et al., 1990), increase of macrophage numbers was prevented and neuropathological features were substantially ameliorated in two CMT1 models (Carenini et al., 2001; Groh et al., 2012), most likely by blocking the impact of macrophages on Schwann cell dedifferentiation (Groh et al., 2015b). In a translational approach, treatment with a CSF-1 receptor (CSF-1R) inhibitor (Elmore et al., 2014) improved genetically mediated neuropathy in two models (Klein et al., 2015) reflecting the clinical relevance of the CSF-1/CSF-1R axis.

Due to a combination of alternative splicing and differential proteolysis, CSF-1 exists in three biologically active, homodimeric isoforms: a membrane-spanning, cell-surface glycoprotein (csCSF-1), and two secreted isoforms, a secreted proteoglycan (spCSF-1) and a secreted glycoprotein (sgCSF-1; Pixley and Stanley, 2004). Both spCSF-1 and sgCSF-1 are present at similar concentrations in the circulation (Price, 1992). However, spCSF is the predominant secreted isoform and has broader signaling function in vivo (Price et al., 1992; Pixley and Stanley, 2004). csCSF-1 regulates tissue macrophage populations (Hiroyasu et al., 2013) and Paneth cells locally (Huynh et al., 2009) and does not significantly contribute to circulating CSF-1 levels (Dai et al., 2004), whereas the secreted isoforms can regulate macrophage populations both locally and systemically. The functions of each of the isoforms have previously been studied in $C s f 1^{o p / o p}$ mice that carry individual Csf1 promoter-first intron-driven transgenes restoring normal tissue-specific and developmental expression of csCSF-1 (TgCS; Dai et al., 2004), spCSF-1 (TgSPP; Nandi et al., 2006), or sgCSF-1 (TgSGP; Nandi et al., 2006), or of all three isoforms (TgC; Ryan et al., 2001). Hemizygous carriers of these transgenes express normal levels of the respective CSF-1 isoforms and correct distinct abnormalities of Csf1 ${ }^{o p / o p}$ mice with different efficacy.

Endoneurial fibroblasts are the major source of CSF-1 in diseased nerves of CMT1 models and patients and are often in direct cell-cell association with macrophages (Groh et al., 2012). We therefore chose to investigate the putatively distinct roles of cellsurface versus secreted isoforms of CSF-1 in a model of CMT1X, connexin32-deficient ( $C x 32$ def $)$ mice. Because homozygous carriers of the available CSF-1 transgenes overexpress the corresponding isoforms (Ryan et al., 2001; Dai et al., 2004; Nandi et al., 2006), we had the additional opportunity to investigate their impact on CSF-1-dependent macrophage activation in Cx32def mice as a reciprocal approach to CSF-1 deficiency. To address these questions we crossbred Cx32wt/Csfl ${ }^{\text {op/+ }}$ and Cx32def/ Csf $1^{o p /+}$ mice with Csf $1^{o p / o p} / \mathrm{TgC}, \mathrm{Csf} 1^{o p / o p} / \mathrm{TgCS}$ or $C s f 1^{o p / o p} / \mathrm{Tg}$ $S P P$ mice to restore or overexpress all $(\mathrm{TgC})$, or distinct ( $\mathrm{TgCS}$ or
TgSPP) isoforms. Here we show that csCSF-1 and spCSF-1 exert opposing roles in macrophage-mediated neural damage in $\mathrm{Cx} 32$ deficient mice.

\section{Materials and Methods}

Animals. Connexin32-deficient (Cx32def: $C \times 32^{-1-}$ or $\left.C \times 32^{-/ y}\right)$ mice (Nelles et al., 1996; Anzini et al., 1997) and wild-type (wt) littermates ( Cx32wt: $\mathrm{C} \times 32^{+/+}$or $C \times 32^{+/ y}$ ) were crossbred with $C s f 1^{\text {op } /+}$ mice bearing the osteopetrotic allele (Yoshida et al., 1990). Both lines were on a uniform C57BL/6N genetic background. Cx32wt/Csf $1^{\text {op/+ }}$ and Cx32def/ $C s f 1^{o p /+}$ mice were then crossbred with $C s f 1^{o p / o p} ; \mathrm{TgC2}$ (Ryan et al., 2001), Csf1 $1^{o p / o p}$; TgCS5 (Dai et al., 2004), or Csf1 ${ }^{o p / o p}$; TgSPP2 (Nandi et al., 2006) transgenic mice on a uniform FVB/NJ genetic background. For each CSF-1 isoform, the particular hemizygous Csf1 transgenic lines used for this study exhibited expression patterns characteristic of the majority of independently derived lines developed for that isoform (Ryan et al., 2001; Dai et al., 2004; Nandi et al., 2006). F1 and F2 generation offspring mice were then intercrossed to acquire homozygously transgenic mice (up to F3 generation). Mice of either sex were euthanized and peripheral nerves were analyzed at the age of 12 months. Cx32wt/Csf1wt $\left(C \times 32^{+/+}\right.$ or $C \times 32^{+/ y} ; C s f 1^{+/+}$or $\left.C s f 1^{o p /+}\right), C x 32 w t / C s f 1 o p\left(C \times 32^{+/+}\right.$or $C x 32^{+/ y}$; $\left.C s f 1^{o p / o p}\right), C x 32 \mathrm{def} / \mathrm{Csf1wt}\left(\mathrm{Cx} 32^{-1-}\right.$ or $C \times 32^{-/ y} ; C s f 1^{+/+}$or $\left.C s f 1^{o p /+}\right)$ and $C \times 32$ def/Csf1op $\left(C \times 32^{-1-}\right.$ or $\left.C x 32^{-1 y} ; C s f 1^{o p / o p}\right)$ mice on F1, F2, and F3 C57BL/6N $\times$ FVB/NJ genetic background showed comparable macrophage and fibroblast numbers, axon counts, and neuropathological phenotypes to mice on a uniform C57BL/6N background in all experiments (cf. Groh et al., 2012). Determination of genotypes was performed using isolated DNA from ear punch biopsies following previously published protocols with conventional PCR and previously described primer pairs or by semiquantitative real-time PCR using SYBR Green PCR Master Mix and Gapdh as internal standard (Applied Biosystems; Carenini et al., 2001; Ryan et al., 2001; Kobsar et al., 2003; Dai et al., 2004; Nandi et al., 2006). Mice were kept in the animal facility of the Department of Neurology under barrier conditions (individually ventilated cages) and all experiments were approved by the local authority (Government of Lower Franconia, Germany).

Immunohistochemistry. For preparation of cryosections, animals were euthanized by asphyxiation with $\mathrm{CO}_{2}$ (according to guidelines by the State Office of Health and Social Affairs Berlin), blood was rinsed with PBS containing heparin, femoral quadriceps nerves were excised, processed as described previously (Groh et al., 2012), and cut into 10- $\mu \mathrm{m}$ thick cross-sections on a cryostat (Leica). Fresh frozen nerve sections were postfixed in acetone $\left(10 \mathrm{~min},-20^{\circ} \mathrm{C}\right)$ and incubated with $5 \% \mathrm{BSA}$ in $0.1 \mathrm{M}$ PBS for $30 \mathrm{~min}$ at room temperature to block unspecific binding sites. Afterward, the respective primary antibodies (rat anti-F4/80, 1:300, Serotec; rat anti-CD34, 1:1000, eBioscience; rat anti-CD86, 1:100, BD Biosciences; rat anti-CD206, 1:2000, Serotec; rabbit anti-CSF-1, 1:300, Santa Cruz Biotechnology) were incubated overnight at $4^{\circ} \mathrm{C}$ in $1 \%$ BSA in $0.1 \mathrm{M}$ PBS and detected by corresponding secondary antibodies (goat anti-rat Cy2, Dianova; goat anti-rat Cy3, 1:300, Dianova; goat anti-rabbit Cy3, 1:300, Dianova; goat anti-rabbit Cy5, 1:500, Dianova; goat anti-rat Cy5, 1:500, Dianova). Macrophages in contact with endoneurial fibroblasts were visualized as previously described (Groh et al., 2012). Nuclei were stained with DAPI (Sigma-Aldrich) and samples were mounted with Aqua-Poly/Mount (Polysciences) and investigated on an Axiophot 2 epifluorescence microscope (Zeiss) or a FluoView FV1000 confocal microscope (Olympus). Only cell profiles containing a DAPI-labeled nucleus were considered for quantification. Form factor $(4 \pi \times$ cell area/ perimeter ${ }^{2}$ ) analysis was performed as previously described (Müller et al., 2007).

Semiquantitative real-time PCR. After rinsing the blood with PBS containing heparin, sciatic nerves were quickly dissected, snap frozen in liquid nitrogen, and stored at $-80^{\circ} \mathrm{C}$ until further processing. Nerves were homogenized (ART-MICCRA D-8, ART Labortechnik) in TRIzol reagent (Invitrogen) and total RNA was isolated according to the manufacturers' guidelines. Concentration and quality of RNA was determined using a BioPhotometer (Eppendorf) and $1 \mu \mathrm{g}$ of RNA was reverse transcribed in a $100 \mu \mathrm{l}$ reaction using random hexamer primers (Applied 


\section{relative Csf1 mRNA expression}

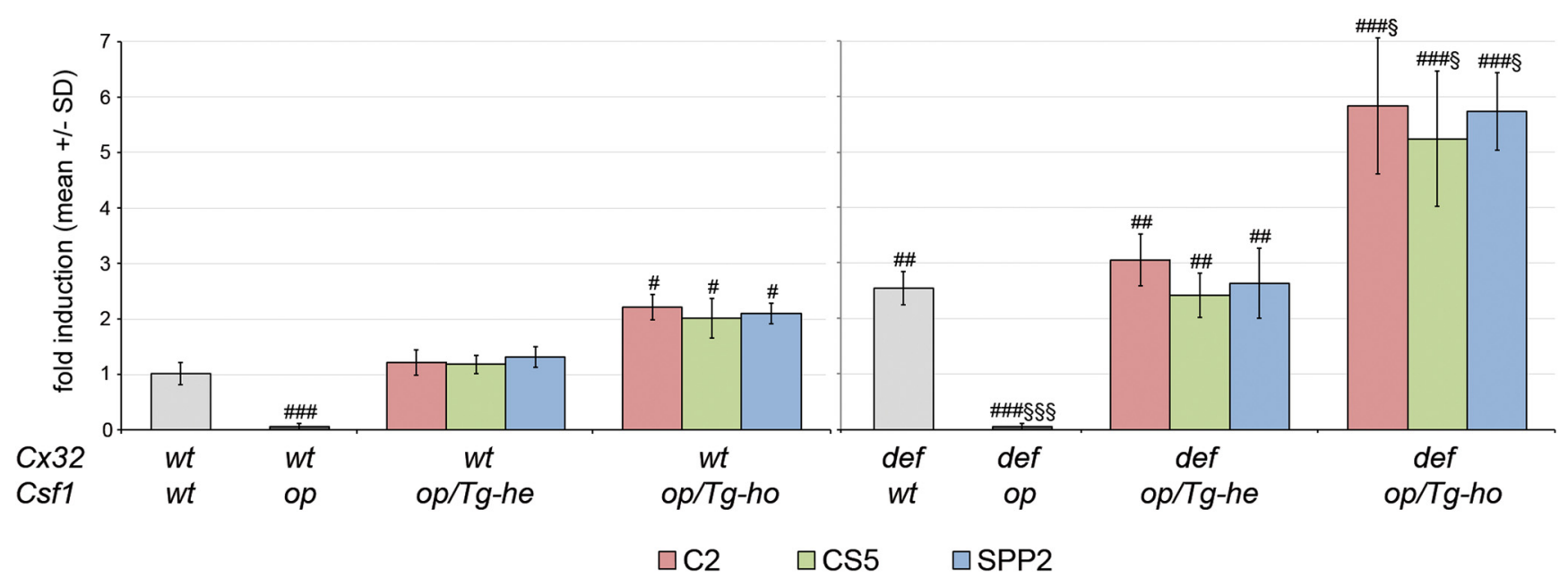

Figure 1. Relative expression of $(s f 1 \mathrm{mRNA}$ in peripheral nerves of isoform-specific transgenic mice. Semiquantitative real-time PCR for Csf1 mRNA normalized to Gapdh as endogenous control in sciatic nerves from 12-month-old CX32wt/Csf1wt, CX32wt/Csf1op, CX32def/Csf1wt, and CX32def/Csf1op mice, as well as nerves from Cx32wt/Csf1op and CX32def/Csf1op mice Tg-he or Tg-ho for C2, CS5, or SPP2. Csf1 mRNA expression was strongly reduced in nerves of Cx32wt/Csf1op and Cx32def/Csf1op mice, but was restored in Tg-he mice and increased in Tg-ho mice in relation to the

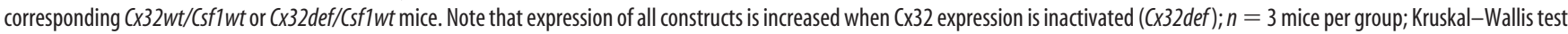

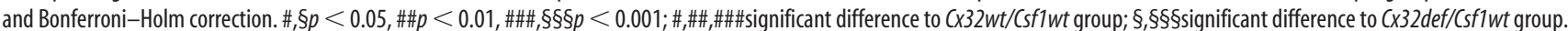

Biosystems). Complementary DNA samples were subsequently analyzed by semiquantitative real-time PCR using predeveloped TaqMan assays (Murine Csf1, Mm00432684_m1; Murine Gapdh as internal standard, Mm99999915_g1) and TaqMan universal PCR master mix (Applied Biosystems) according to the manufacturers' guidelines.

Morphometric analysis by electron microscopy. Femoral quadriceps nerves and lumbar ventral roots were processed for light and electron microscopy as previously described (Groh et al., 2012). Briefly, mice at the age of 12 months were transcardially perfused with $4 \%$ paraformaldehyde and $2 \%$ glutaraldehyde in $0.1 \mathrm{~m}$ cacodylate buffer. Dissected nerves were postfixed in the same solution overnight at $4^{\circ} \mathrm{C}$, followed by osmification, dehydration, and embedding in Spurr's medium. Semithin $(0.5 \mu \mathrm{m})$ cross-sections were stained with alkaline methylene blue for light microscopy and ultrathin sections $(70 \mathrm{~nm})$ were mounted to copper grids and counterstained with lead citrate for electron microscopy. Morphometric analysis was performed with a ProScan Slow Scan CCD camera mounted to a Leo $906 \mathrm{E}$ electron microscope (Zeiss) and corresponding software iTEM (Soft Imaging System). Multiple image alignments were acquired and characteristic pathological alterations were quantified in relation to the total number of myelin competent axons in whole nerve cross-sections.

Statistical analysis. All quantifications were performed by investigators unaware of the genotypes of the respective mice. Data are represented as mean \pm SD. Statistical analyses were performed using PASW Statistics 18 (SPSS, IBM) software. Data were controlled for normal distribution by Shapiro-Wilk test and group differences were tested either by one-way ANOVA followed by Tukey's post hoc test (parametric) or Kruskal-Wallis test with Bonferroni-Holm correction (nonparametric). Significant differences were indicated as follows: ${ }^{\star}, \#, \$ p<0.05,{ }^{*}$, $\# \#, \$ \$ p<0.01$, ${ }^{* * *}$,\#\#\#,\$\$\$p<0.001;,*,**,** significant difference between corresponding groups; \#,\#\#\#\#\# significant difference to Cx32wt/Csf1wt group; $₫, \$ \$, \$ \$ \$$ significant difference to $C x 32 \mathrm{def} / C s f 1 w t$ group.

\section{Results \\ Impact of distinct CSF-1 isoforms on endoneurial macrophages and fibroblasts}

At first, we investigated the expression of Csf1 mRNA in sciatic nerves of wild-type (Cx32wt/Csfiwt) $\left(C \times 32^{+/+}\right.$or $C \times 32^{+/ y}$; $\left.C s f 1^{+/+}\right)$, connexin32-deficient $(C \times 32$ def/Csflwt $)\left(C \times 32^{-1-}\right.$ or $\left.C \times 32^{-/ y} ; C s f 1^{+/+}\right)$, CSF-1-deficient (Cx32wt/Csflop) $\left(C \times 32^{+/+}\right.$or $\left.C \times 32^{+/ y} ; C s f 1^{o p / o p}\right)$, or Cx32 and CSF-1 double- deficient (Cx32def/Csf1op) (Cx32 $2^{-1-}$ or $\left.C \times 32^{-/ y} ; C s f 1^{o p / o p}\right)$ mice using a gene expression assay at the exon boundary $4-5$ in the protein coding region. Cx32def/Csflwt myelin mutant mice showed an $\sim 2.5$-fold increase in Csf1 mRNA expression compared with $C \times 32 w t / C s f 1 w t$ mice at the age investigated, whereas Csf1 mRNA was barely detectable in Cx32wt/Csflop and Cx32def/Csflop mice (Fig. 1).

We then investigated Cx32wt/Csflop and Cx32def/Csflop mice either hemizygously (he) or homozygously (ho) transgenic (Tg) for the distinct constructs (Ryan et al., 2001; Dai et al., 2004; Pixley and Stanley, 2004; Nandi et al., 2006) that drive expression of different CSF-1 isoforms. Cx32wt/Csflop mice hemizygous for $\mathrm{TgC2}$, TgCS5, or TgSPP2 showed restored mRNA expression levels similar to wild-type mice, whereas Cx32wt/Csflop mice homozygous for $\mathrm{TgC} 2, \mathrm{TgCS} 5$ or $\mathrm{TgSPP} 2$ showed an approximately twofold increase in Csf1 mRNA expression. Cx32def/Csf1op mice hemizygous for $\mathrm{TgC} 2, \mathrm{TgCS} 5$, or $\mathrm{TgSPP} 2$ showed a twofold to threefold increase as also seen in Cx32def/Csflwt mice and Cx32def/Csf1op mice homozygous for TgC2, TgCS5, or TgSPP2 showed a fivefold to sixfold increased expression (Fig. 1). This demonstrates that Csf1 mRNA expression in peripheral nerves of Csflop mice is restored in hemizygously transgenic mice as seen previously in other tissues (Ryan et al., 2001) and increased (approximately twofold) in homozygously transgenic mice regardless of the transgenic construct. Furthermore, $C \times 32$ deficiency increases the expression of CS5 and SPP2 transgene-derived Csf 1 mRNA (generating 1 specific isoform each) as similarly observed in Cx32def/Csflwt mice, as well as C2 transgene-derived mRNA generating all three isoforms (Ryan et al., 2001; Dai et al., 2004; Pixley and Stanley, 2004; Nandi et al., 2006).

As a next step, we investigated the number of macrophages in femoral quadriceps nerves of Cx32wt/Csf1wt, Cx32def/Csflwt, $C \times 32 w t / C s f 1 o p$, and Cx32def/Csflop mice (Fig. 2). Corroborating previous findings (Groh et al., 2012), Cx32wt/Csf1wt, Cx32wt/ Csf1op, and Cx32def/Csflop mice showed similar macrophage numbers, with all of the mutant mice (either for $C \times 32$ and/or Csf1) being in the range of resident macrophages seen in Cx32wt/ 


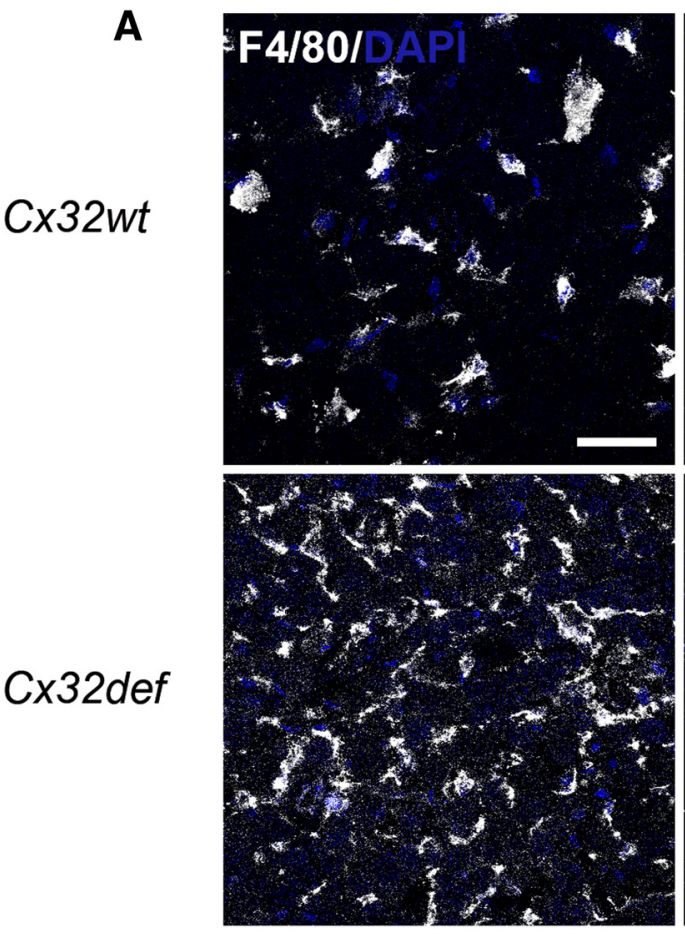

Csf1op/Tg-ho $\square \mathrm{C} 2$
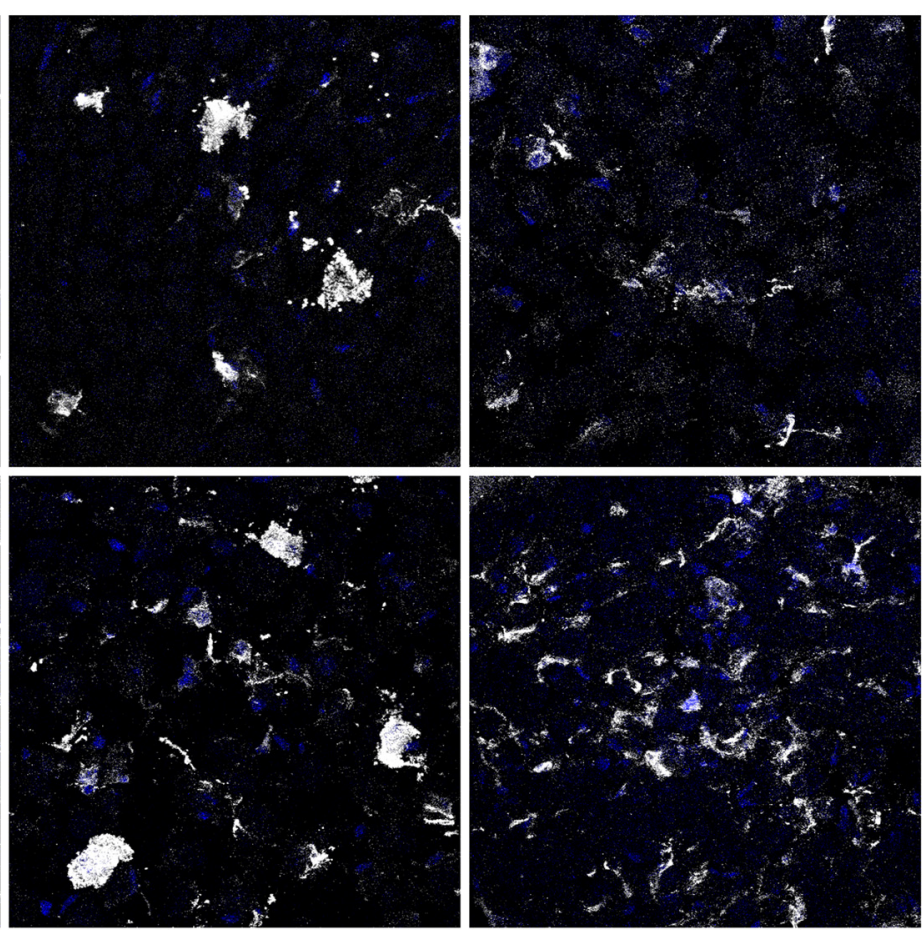

$\square$ CS5

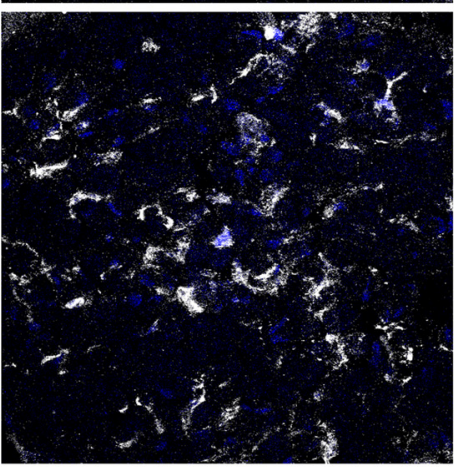

$\square$ SPP2

B

F4/80+ Macrophages

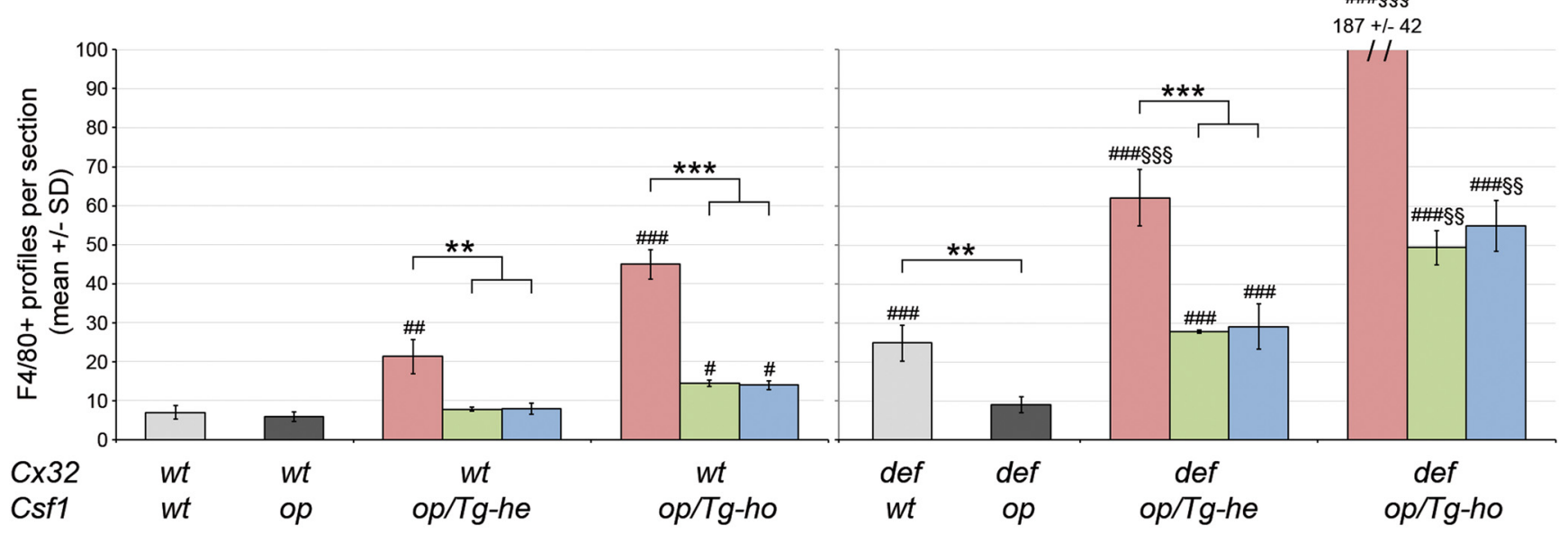

$\square \mathrm{C} 2 \quad \square \mathrm{CS} 5 \quad \square \mathrm{SPP} 2$

Figure 2. Cell-surface and secreted CSF-1 isoforms regulate macrophage numbers and morphology in peripheral nerves of $C x 32$ wt and $C \times 32$ def mice. $A$, Immunohistochemistry of F4/80-positive macrophages in cross-sections of femoral quadriceps nerves from 12-month-old CX32wt/Csf1op and CX32def/Csf1op mice Tg-ho for C2, CS5, or SPP2. TgCS5 expression resulted in bigger and less ramified appearance of macrophages compared with macrophages in TgSPP2- or TgC2-expressing mice. Scale bar, $30 \mu \mathrm{m}$. B, Quantification of F4/80-positive macrophages in quadriceps nerves from 12-month-old CX32wt/Csf1wt, CX32wt/Csf1op, CX32def/Csf1wt, and CX32def/Csf1op mice, as well as nerves from CX32wt/Csf1op and Cx32def/Csf1op mice Tg-he or Tg-ho for C2, CS5, or SPP2. Either TgCS5 or TgSPP2 restored macrophage recruitment in CX32def/Csf1op mice when hemizygously expressed and enhanced macrophage recruitment in Cx32wt/Csf1op and Cx32def/Csf1op mice when homozygously expressed. TgC2 expression enhanced macrophage recruitment in all conditions; $n=4$ mice per group; one-way ANOVA and Tukey's post hoc tests. \#p $<0.05$, ${ }^{* *}, \# \#, \S \S p<0.01,{ }^{* * *}, \# \# \#, \S \S \S p<0.001 ;{ }^{* * * * * *}$ significant difference between corresponding groups; \#\#,\#\#\#significant difference to Cx32wt/Csf1wt group; $\S \S, \S \S \S s i g n i f i c a n t$ difference to $C \times 32$ def/Csfiwt group.

Csf1wt mice. By contrast, and as published previously (Groh et al., 2012), Cx32def/Csf1wt myelin mutant mice showed, as opposed to Cx32def/Csf1op mice, approximately a triplication of macrophage numbers at the age investigated.

Cx32wt/Csf1op mice hemizygous for TgCS5 or TgSPP2 did not show a change in macrophage numbers, as opposed to Cx32wt/Csf1op mice that are hemizygously transgenic for $\mathrm{TgC} 2$ and expressing all three isoforms (threefold increase). Cx32wt/ Csf1op mice homozygous for TgCS5 or TgSPP2 showed a mild (twofold) elevation of macrophage numbers, whereas CX32wt/ Csf1op mice homozygous for TgC2 exhibited a robust elevation by a factor of approximately six. Cx32def/Csf1op mutants hemizygously expressing TgCS5 or TgSPP2 mimicked the approximate triplication of macrophage numbers seen in Cx32def/Csflwt 
mice, whereas hemizygous $\mathrm{TgC} 2$ expression caused an approximately ninefold increase in macrophage numbers. Macrophage numbers in Cx32def/Csflop mice were further enhanced by homozygous expression of TgCS5 or TgSPP 2 and again most prominently increased by homozygous TgC2. Thus TgCS5 or TgSPP2 always caused a comparable increase in macrophage numbers, whereas $\mathrm{TgC}$, driving expression of all three isoforms, always caused the most prominent elevation (Fig. 2). This demonstrates that both spCSF-1 and csCSF-1 are equally able to increase macrophage numbers in peripheral nerves. Additionally, overexpression of CSF-1 (Tg-heC2; Tg-hoC2; Tg-hoCS5; Tg-hoSPP2) enhances macrophage recruitment not only in Cx32def mice but also in Cx32wt mice.

Interestingly, restoring the expression of (or overexpressing) distinct CSF-1 isoforms differentially affected endoneurial macrophage morphology. This was demonstrated by analysis of the form factor as a measure of macrophage circularity (maximum form factor: 1 for a perfect circle) as previously described (Müller et al., 2007). Macrophages in nerves of TgCS5-expressing Cx32wt/Csf1op or Cx32def/Csf1op mice displayed a rounder and less ramified appearance reflected by a higher form factor $(0.54 \pm$ 0.16 and $0.61 \pm 0.16$, respectively) compared with those in nerves of the corresponding TgSPP 2 mice $(0.24 \pm 0.08$ and $0.24 \pm 0.06)$. Re-expressing all isoforms ( $\mathrm{TgC} 2)$ resulted in a mixed population with both slender but also rounded macrophages reflected by an intermediate form factor $(0.44 \pm 0.19$ and $0.54 \pm 0.18)$.

Although csCSF-1 and spCSF-1 had a similar influence on macrophage numbers under all genetic conditions, their impact on the expression of distinct markers for macrophage activation was completely different. In both Cx32wt/Csf1wt (data not shown) and in Cx32def/Csflwt mice (Fig. 3), $\sim 50 \%$ of all macrophages were positive for the activation marker CD86 and $\sim 40 \%$ were positive for CD206. Also, in both Cx32wt/Csf1op (data not shown) and Cx32def/Csflop mice (Fig. 3), completely lacking CSF-1, this relationship was not altered. However, hemizygosity (data not shown) and homozygosity for TgCS5 led to strongly decreased expression of CD86 and CD206 on macrophages, whereas hemizygosity (data not shown) and homozygosity for TgSPP2 robustly elevated the expression of both markers on endoneurial macrophages in Cx32wt/Csf1op (data not shown) and Cx32def/Csflop mice (Fig. 3). Thus, csCSF-1 robustly inhibits macrophage activation, whereas spCSF-1 enhances macrophage activation in peripheral nerves.

As we have previously shown that endoneurial fibroblasts are a major source of CSF-1 in the peripheral nerve and that many of these cells are in close contact with macrophages (Groh et al., 2012), we investigated the number of endoneurial fibroblasts in the mutants (Fig. 4). In Cx32wt/Csf1op mice and in all Cx32wt/ Csflop mice with hemizygous expression of the CSF-1 transgenes, fibroblast numbers were comparable to those of $C \times 32 w t /$ Csf1wt mice. In Cx32wt/Csf1op mice, homozygosity for TgC2 or TgCS5 resulted in a duplication of the fibroblasts, whereas homozygosity for TgSPP2 did not elevate the fibroblast numbers. $C \times 32$ deficiency resulted in duplication of fibroblast numbers in Csflwt and Csflop mice, and also when the CSF-1 transgenes were hemizygously expressed. When homozygously expressed, TgSPP2 again did not elevate fibroblast numbers in Cx32def mice, as opposed to homozygously expressed TgC2 or TgCS5. Despite the differential impact of homozygous TgCS5 and TgSPP2 on fibroblast numbers, their overexpression did not influence the percentage of macrophage-fibroblast contacts ( $~ 60-70 \%$ of macrophages were in contact with fibroblasts showing no significant difference). These data suggest that fibro-
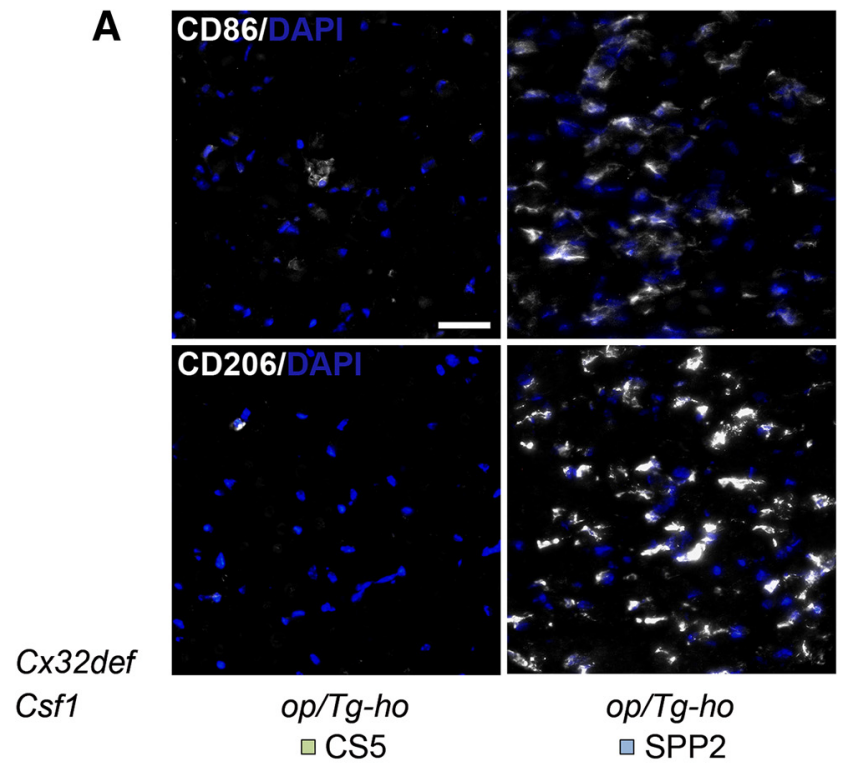

B

Macrophage activation

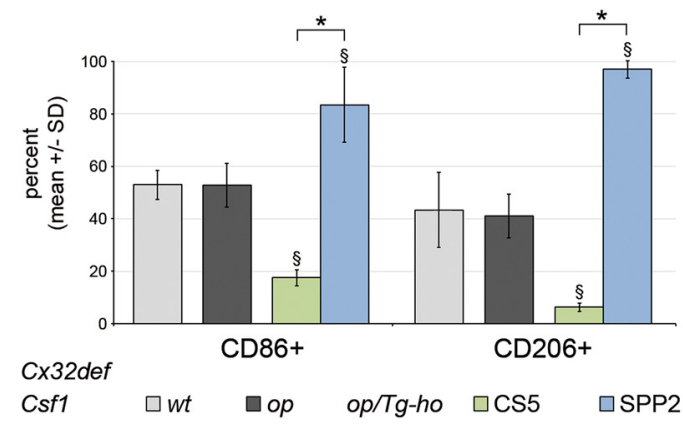

Figure 3. Differential effects of csCSF-1 and spCSF-1 on macrophage activation in peripheral nerves of $C$ 32 def mice. $A$, Immunohistochemistry of activated CD86-positive (top) and CD206positive (bottom) macrophages in cross-sections of femoral quadriceps nerves from 12-monthold CX32def/Csf1op mice Tg-ho for CS5 or SPP2. Scale bar, $30 \mu \mathrm{m}$. B, Quantification of activated macrophages as percentages of immunoreactive profiles related to total macrophage numbers, based on CD86/F4/80 and CD206/F4/80 double-labeling. cSCSF-1 expression reduced macrophage activation, whereas spCSF-1 expression enhanced it; $n=4$ mice per group; KruskalWallis test and Bonferroni-Holm correction. ${ }^{*}, \$ p<0.05$; * significant difference between corresponding groups; §significant difference to $C \times 32$ def/Csf1wt group.

blast numbers are determined by two factors: (1) Cx32 deficiency and (2) csCSF-1 (also significantly restored by the TgC2 construct; Ryan et al., 2001).

To determine whether the different isoforms of CSF-1 are expressed by the same cell types in mutant nerves we performed triple immunohistochemistry using polyclonal antibodies against CSF-1 in combination with macrophage and fibroblast markers (Fig. 5). In Cx32def/Csf1wt mice, CSF-1 immunoreactivity was detected in $33 \%$ of fibroblasts and $7 \%$ of macrophages, corroborating previous observations using different techniques (Groh et al., 2012). In contrast, $7 \%$ of fibroblasts and $26 \%$ of macrophages showed CSF-1 immunoreactivity in Cx32def/ Csf1op mice homozygously transgenic for CS5. Homozygous expression of TgSPP2 resulted in CSF-1 immunoreactivity in 48\% of fibroblasts and $9 \%$ of macrophages (Fig. $5 A$ ). Similar results were obtained in mice with hemizygous expression of the respective constructs (data not shown). These observations suggest that endoneurial fibroblasts mostly express the spCSF-1, whereas csCSF-1 is mostly expressed by endoneurial macrophages. In ad- 


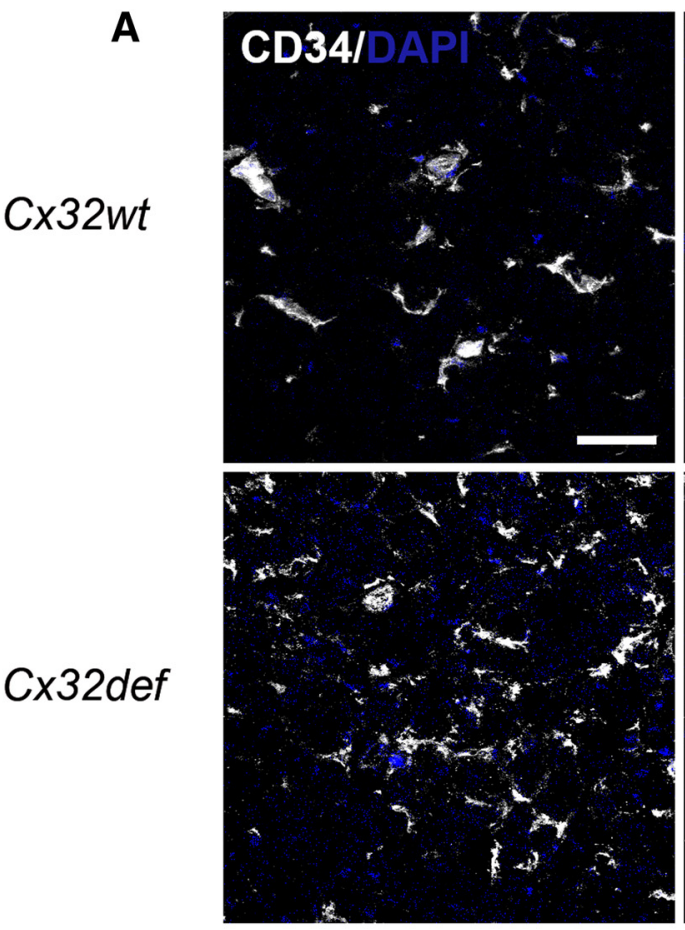

Csf1op/Tg-ho $\square \mathrm{C} 2$

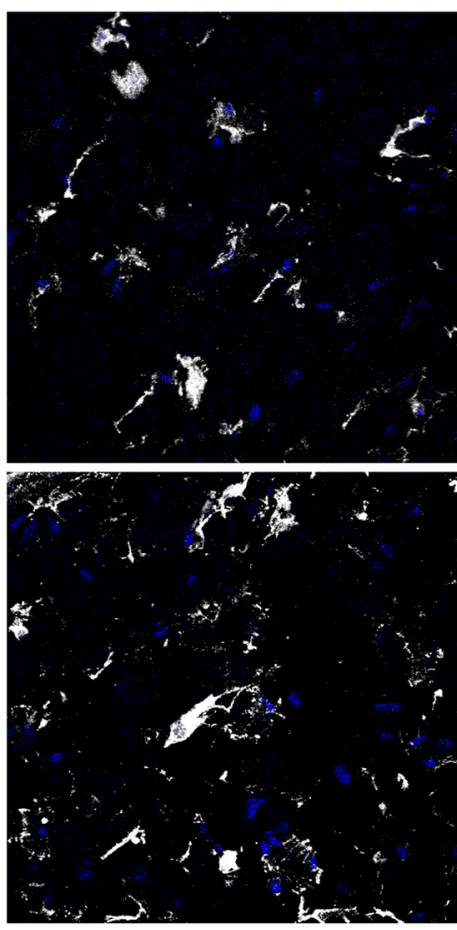

$\neg$ CS5

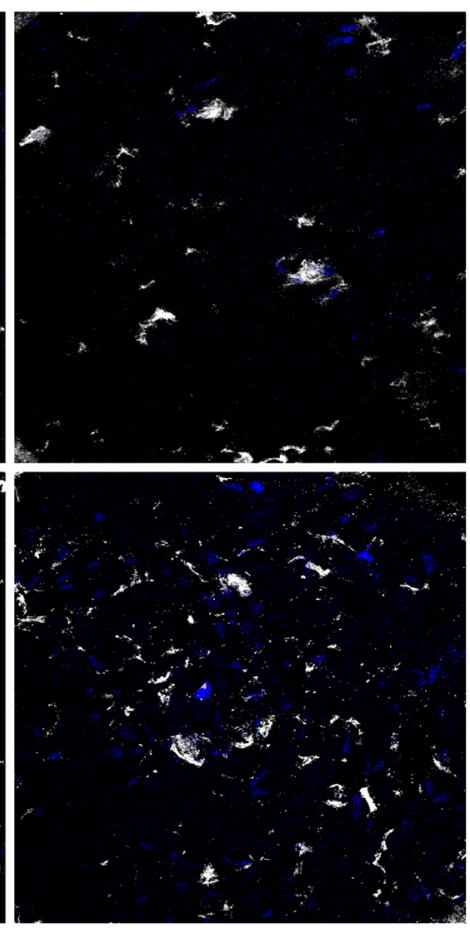

$\square$ SPP2

B

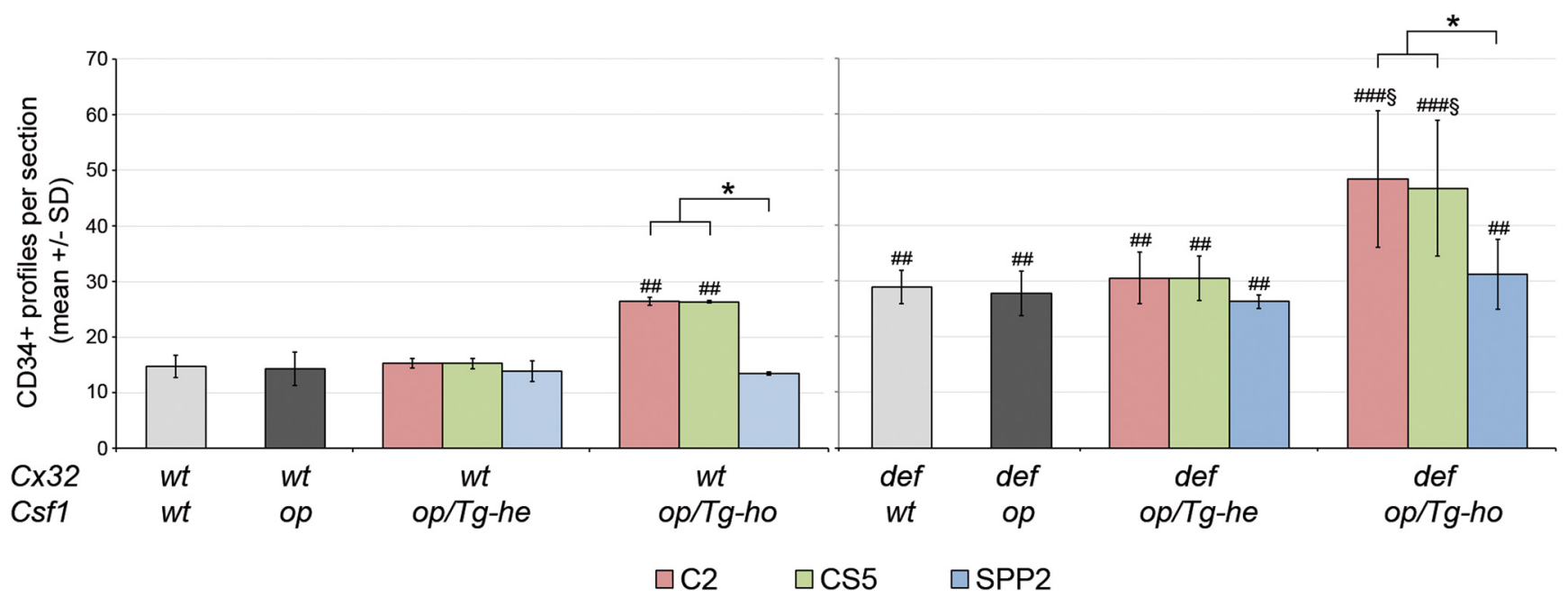

Figure 4. Differential effects of cSCSF- 1 and spCSF- 1 on fibroblast numbers in peripheral nerves of $C x 32 w t$ and $C x 32$ def mice. $A$, Immunohistochemistry of CD34-positive fibroblasts in cross-sections of femoral quadriceps nerves from 12-month-old Cx32wt/Csf1op or Cx32def/Csf1op mice Tg-ho for C2, CS5, or SPP2. Scale bar, $30 \mu \mathrm{m}$. B, Quantification of CD34-positive fibroblasts in quadriceps nerves from 12-month-old Cx32wt/Csf1wt, Cx32wt/Csf1op, Cx32def/Csf1wt, or Cx32def/Csf1op mice, as well as nerves from Cx32wt/Csf1op or Cx32def/Csf1op mice Tg-he or Tg-ho for C2, CS5, or SPP2. TgC2 or TgCS5, but not TgSPP2 increased fibroblast numbers in Cx32wt/Csf1op and Cx32def/Csf1op mice when homozygously expressed; $n=$ 4 mice per group; one-way ANOVA and Tukey's post hoc tests. *,$\$ p<0.05$, \#\#p $<0.01$, \#\#\# $<0.001$; ${ }^{*}$ significant difference between corresponding groups; \#\#\#\#\#significant difference to $C \times 32 w t / C 5 f 1 w t$ group; §significant difference to $C x 32$ def/Csf1wt group.

dition, the large majority of CSF-1-expressing fibroblasts were detected in contact with macrophages, whereas CSF-1expressing macrophages were not preferentially associated with fibroblasts (Fig. 5B). In combination with the observations on activation markers above, these results argue for: (1) a juxtacrine activation of macrophages by fibroblasts that secrete the spCSF-1 and (2) for an autocrine inhibition of macrophage activation by the csCSF-1.
Impact of CSF-1 isoforms on pathological alterations of peripheral nerves

As CSF-1 has been identified as robust disease mediator of genetically caused neuropathies (Carenini et al., 2001; Groh et al., 2012), we tested the impact of CSF-1 isoforms on nerve integrity of $C \times 32 w t$ and $C \times 32 d e f$ mice (Figs. 6, 7). In general, we detected an influence of CSF-1 isoforms on the overall size of crosssectioned peripheral nerves from Cx32wt mice (Table 1). 


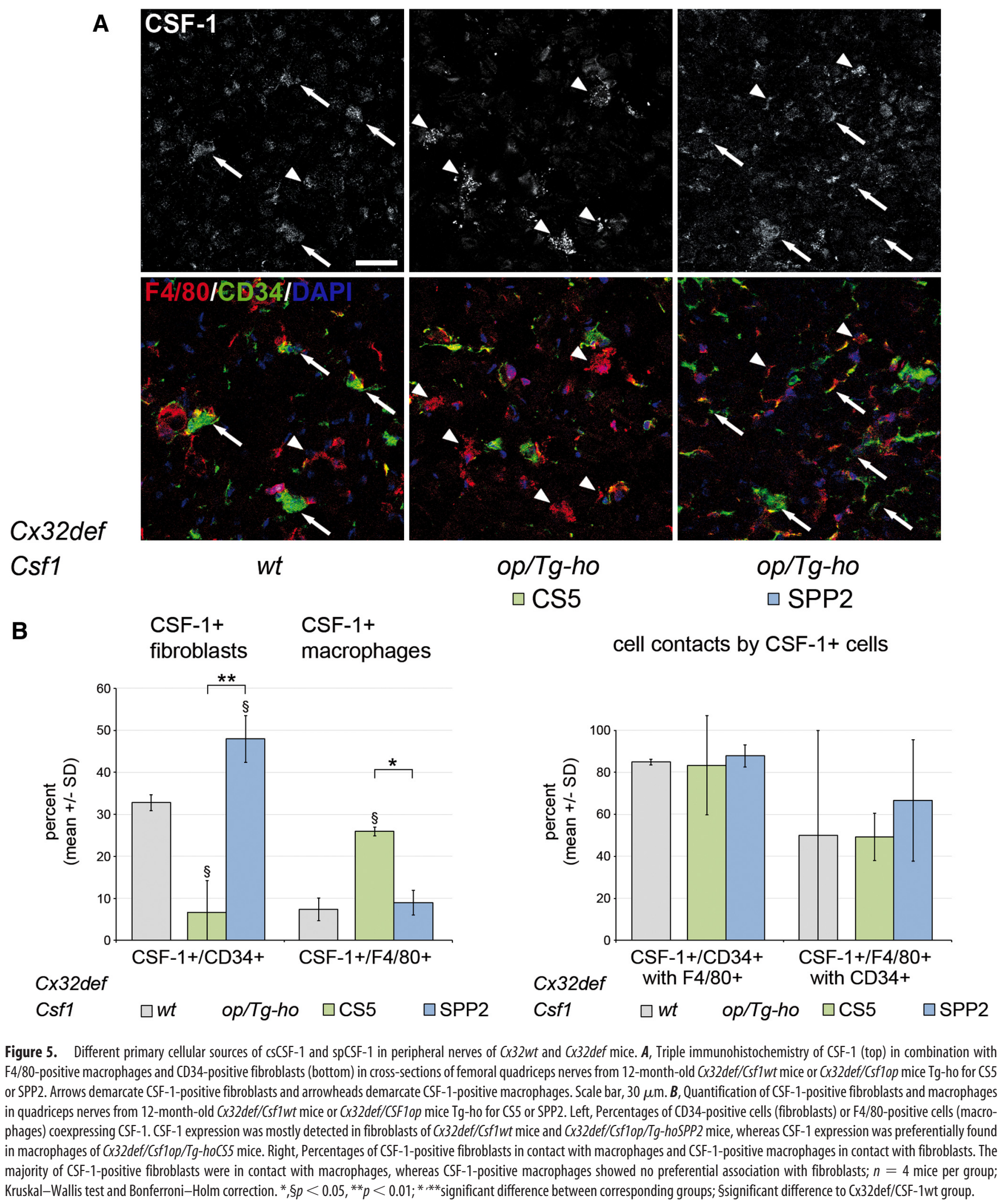

Whereas the perimeter of femoral quadriceps nerves of Cx32wt/ Csf1wt mice was $\sim 800 \mu \mathrm{m}$, those of nerves from Cx32wt/Csf1op mice were reduced by $10 \%$. TgC2 or TgCS5 restored nerve size in Cx32wt/Csf1op mice to wt levels when hemizygously expressed and increased nerve size when homozygously expressed. In contrast, TgSPP2 was unable to fully restore nerve size, even when homozygously expressed. Similar results were obtained in Cx32def mice (data not shown).

In Cx32wt/Csf1op mice, only homozygous TgC2 or TgSPP2 expression caused very mild, but significant changes and only in myelin integrity (Fig. 6). In Cx32def/Csflwt mice, neuropathological changes, such as myelin abnormalities (Fig. 6A), periax- 

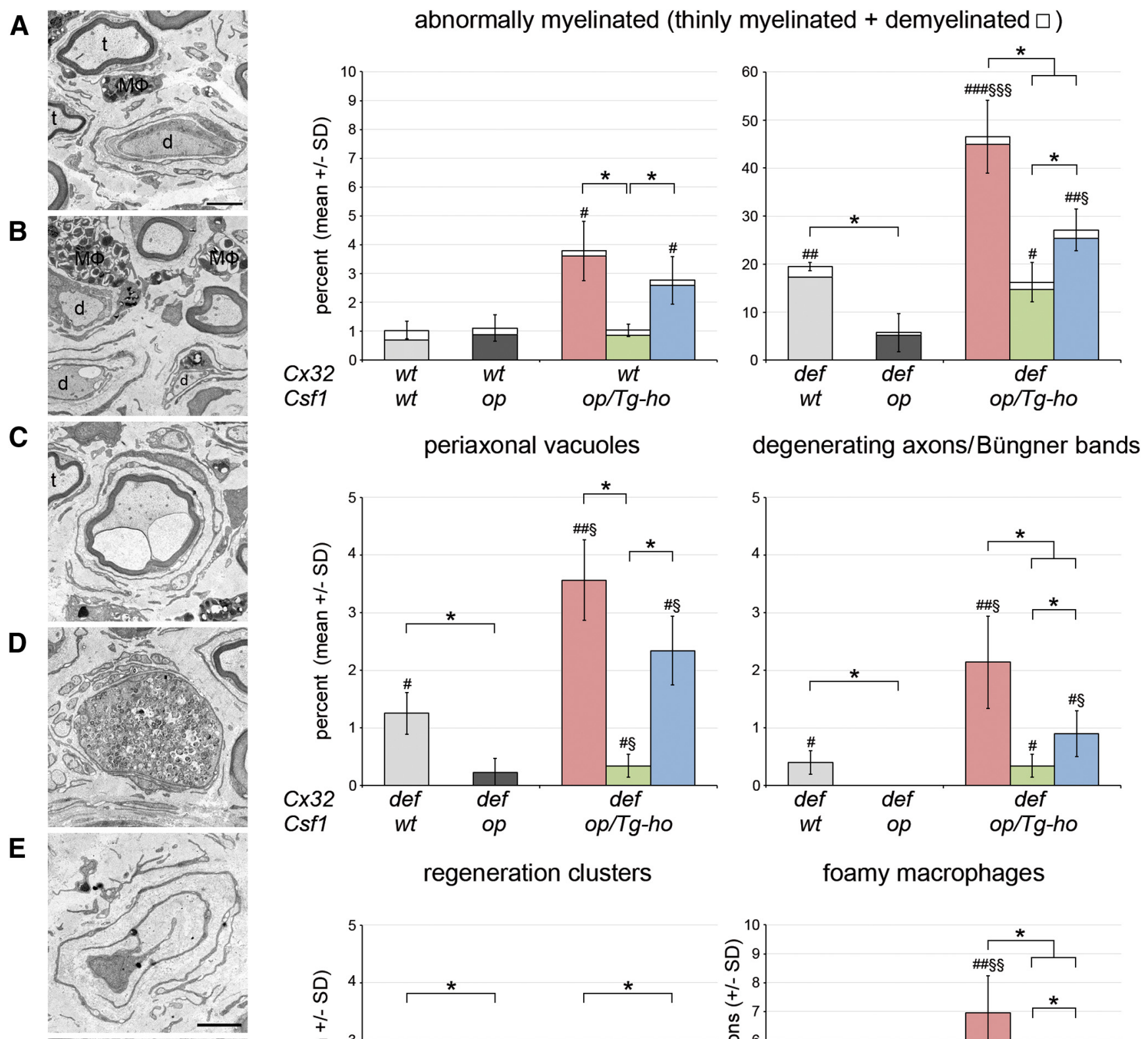

foamy macrophages

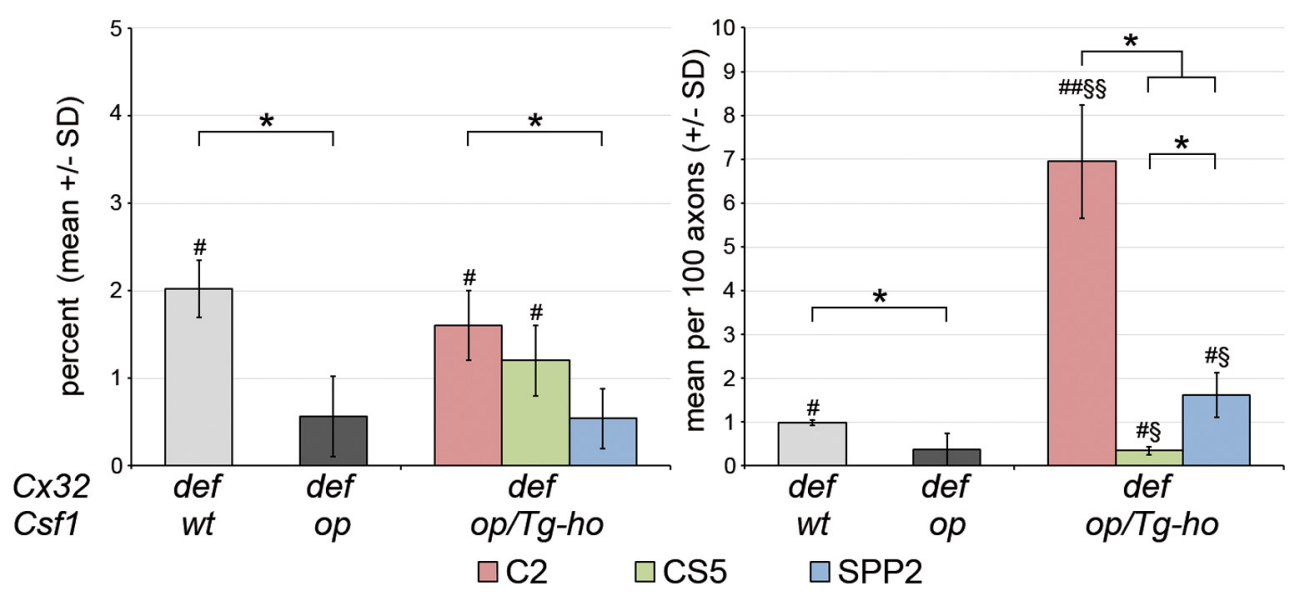

Figure 6. Differential effects cSCSF-1 and spCSF-1 on myelinopathic and axonopathic alterations in femoral quadriceps nerves of (x32def mice. Representative micrographs of (A, $\boldsymbol{B})$ thinly myelinated $(t)$ and demyelinated $(d)$ axons and foamy macrophages $(M \Phi),(\boldsymbol{C})$ periaxonal vacuoles, $(\boldsymbol{D})$ degenerating axons, $(\boldsymbol{E})$ Büngner bands, and $(\boldsymbol{F})$ regeneration clusters by electron microscopy of femoral quadriceps nerves from 12-month-old XX32def/Csf1op/Tg-hoC2 or Cx32def/Csf1op/Tg-hoSPP2 mice. Scale bars, $2 \mu$ m. Morphometric quantification of the these parameters in femoral quadriceps nerves of 12-month-old CX32wt/Csf1wt, Cx32wt/Csf1op, Cx32def/Csf1wt, or Cx32def/Csf1op mice, as well as nerves from Cx32wt/Csf1op or Cx32def/Csf1op mice Tg-ho for C2, CS5, or SPP2 (middle and right). The formation of alterations in myelin integrity (abnormally myelinated axons) in Cx32wt/Csf1op and Cx32def/Csf1op mice was significantly enhanced by homozygous $\operatorname{TgC2}$ or TgSPP2 expression, but not by homozygous $\operatorname{TgCS} 5$ expression. Note the different scaling of the $y$-axes for $C \times 32 w t$ and $C x 32 d e f$ mice. Other pathological alterations were not detected in all Cx32wt groups. Axonopathic alterations and numbers of foamy macrophages in Cx32def/Csf1op mice were also significantly enhanced by homozygous TgC2 or TgSPP2 expression, but not by homozygous TgCS5 expression; $n=4$ mice per group; Kruskal-Wallis test and Bonferroni-Holm correction. * \#, $\$ p<<$


to $C \times 32$ def/Csfiwt group. 

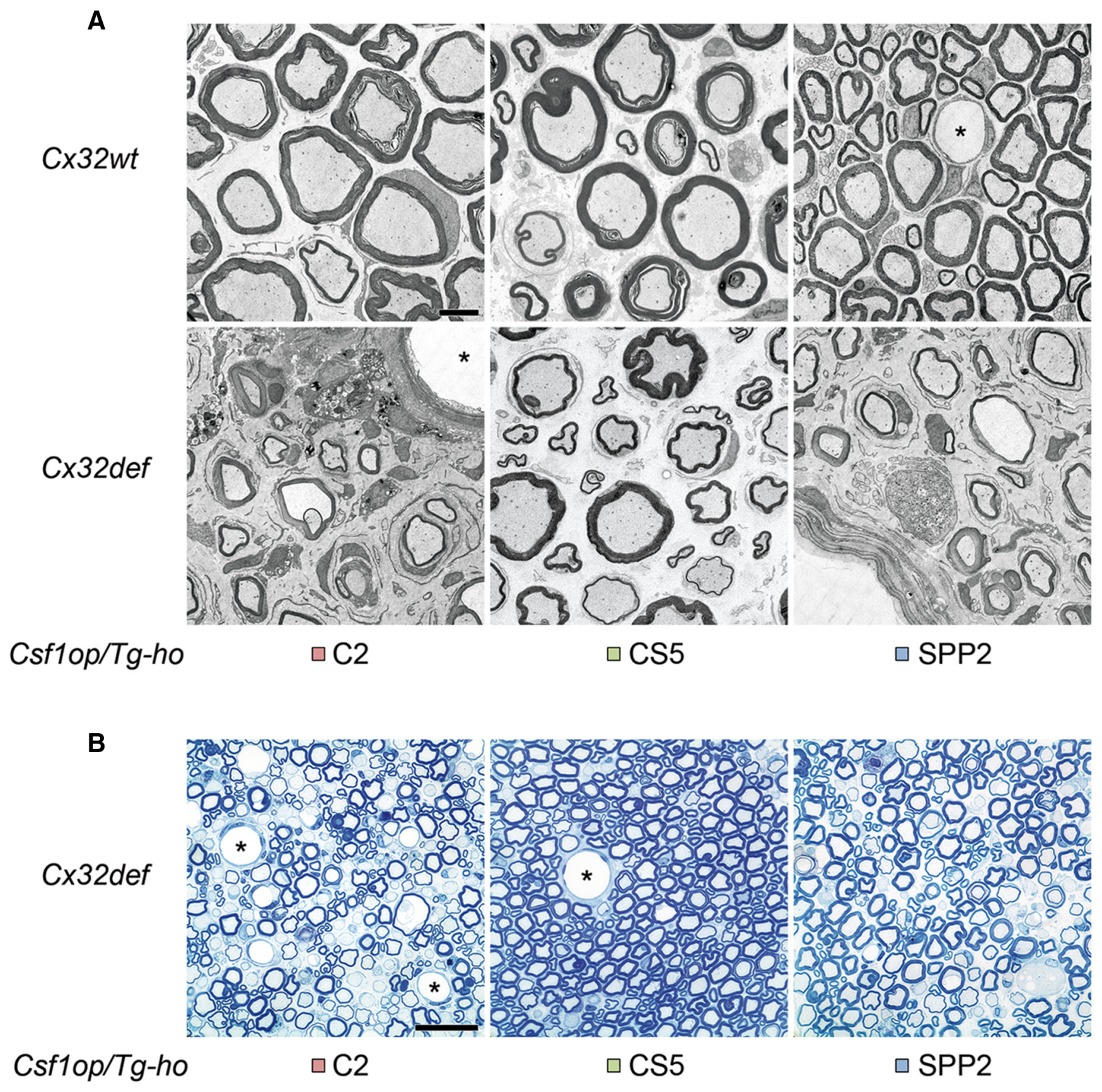

Figure 7. Differential effects of cSCSF-1 and spCSF-1 on pathological alterations in femoral quadriceps nerves and lumbar ventral roots of $C \times 32$ def mice. $A$, Electron microscopy of representative ultrathin sections of femoral quadriceps nerves and (B) light microscopy of representative semithin sections of lumbar ventral roots from 12-month-old CX32wt/Csf1op or CX32def/Csf1op mice Tg-ho for C2, CS5, or SPP2. Note prominent pathological alterations, especially in Cx32def/Csf1op/Tg-hoC2 and Cx32def/Ssf1op/Tg-hoSPP2 mice, such as abnormally myelinated (thinly myelinated and demyelinated) axons, onion bulbs, periaxonal vacuoles, degenerating axons, and regeneration clusters. Such features were less frequent in $\mathrm{Cx} 32 \mathrm{def} / \mathrm{Cs} f 10 \mathrm{op} / \mathrm{Tg}$-hoCS5 mice and rarely detected in all CX32wt genotype groups. Asterisks demarcate blood vessels. Scale bars: $A, 5 \mu \mathrm{m} ; \boldsymbol{B}, 20 \mu \mathrm{m}$. Bottom right micrograph in $A$ is also shown at higher-magnification in Figure $6 D$.

Table 1. Differential effects of csCSF-1 and spCSF-1 on nerve perimeter

\begin{tabular}{|c|c|c|c|c|c|c|c|c|}
\hline & \multicolumn{8}{|l|}{ Csf1 } \\
\hline & \multirow[b]{2}{*}{$w t$} & \multirow[b]{2}{*}{$o p$} & \multicolumn{2}{|l|}{$o p / T g C 2$} & \multicolumn{2}{|l|}{ op/TgCS5 } & \multicolumn{2}{|l|}{ op/TgSPP2 } \\
\hline & & & he & ho & he & ho & he & ho \\
\hline $\begin{array}{l}\text { Quadriceps nerve } \\
\text { perimeter, } \mu \mathrm{m} \\
\text { (mean } \pm \mathrm{SD} \text { ) }\end{array}$ & $800.37 \pm 33.90$ & $721.49^{\#} \pm 44.10$ & $820.37 \pm 72.65$ & $1047.46^{\#} \pm 54.83$ & $822.68 \pm 15.30$ & $1152.14^{\#} \pm 52.31$ & $736.47^{\#} \pm 51.01$ & $765.66 \pm 65.81$ \\
\hline
\end{tabular}

CSF-1 deficiency (Csf1op) results in reduced quadriceps nerve perimeter on a (x32wt background. Hemizygous (he) expression of $\operatorname{TgC2}$ or $\mathrm{TgCS} 5$ restored nerve perimeter, whereas homozygous (ho) expression of TgC2 and TgCS5 increased nerve perimeter. By contrast, TgSPP2 expression did not fully restore normal nerve size, even when homozygously expressed; $n=4$ mice per group; Kruskal-Wallis test and Bonferroni-Holm correction. \#p < 0.05 , \#significant difference to $C x 32 w t /$ CSF-1wt group. 
Table 2. Summary showing the effects of different Csf1 transgenes on peripheral nerve phenotypes in Cx32wt and Cx32def mice



0, Normal wild-type level; + , increase; the relative increase (based on quantitative data) is indicated by the number of symbols; - , decrease; the relative decrease (based on quantitative data) is indicated by the number of symbols; *, an increase is observed or further enhanced in homozygously transgenic mice; F, CSF-1 immunoreactivity of fibroblast; M, CSF-1 immunoreactivity of macrophages.

onal vacuoles (Fig. $6 C$ ) and signs of axonal perturbation (Fig. $6 D, E)$ were robust, as opposed to Cx32def/Csflop doublemutants, corroborating previous results (Groh et al., 2012). In Cx32def/Csf1op mice compared with Cx32def/Csf1wt mice, all neuropathic changes were substantially aggravated by homozygous expression of TgC2 and TgSPP2, whereas neuropathic changes were not as robust in Cx32def/Csf1op mice with homozygous expression of the TgCS5 (Figs. 6, 7A). Generally, the frequency of foamy macrophages (Fig. 6B) was similarly influenced by the CSF-1 isoforms as the neuropathic features. As already described (Groh et al., 2012), the occurrence of regeneration clusters (Fig. $6 F$ ) was robust in Cx32def/Csflwt mice, but rare in Cx32def/Csf1op double-mutants. Similar low numbers of regeneration clusters were seen in Cx32def/Csflop/ TgSPP2 mice, whereas homozygous TgC2 or TgCS5 mildly increased the numbers of regeneration clusters. We also observed a similar impact of the different CSF-1 isoforms on neuropathic alterations in lumbar ventral roots of $C \times 32 \mathrm{def}$ mice (Fig. $7 B$ ). Hemizygous expression of the transgenic constructs in Cx32def/Csf1op mice resulted in similar effects, but only restored and did not aggravate neuropathic alterations beyond levels of Cx32def/Csf1wt mice in case of TgSPP2 (data not shown). In summary, expression of spCSF-1 is more detrimental to neural integrity (severe demyelination, axonal damage, little regeneration) than expression of csCSF-1 (milder demyelination, less axonal damage, more regeneration). Moreover, enhancing macrophage recruitment and activation by overexpressing all CSF-1 isoforms ( Tg-heC2, TghoC2), or spCSF-1 (Tg-hoSPP2), significantly aggravates neuropathy in Cx32def mice, corroborating the important disease-modifying impact of secondary inflammation. Our findings regarding the effects of the distinct transgenic constructs are summarized in Table 2.

\section{Discussion}

Previous studies using Csflop mice intercrossed with POhet and Cx32def myelin mutant mice clearly identified CSF-1 as a robust and sustained amplifier of the primarily genetically caused neuropathies (Carenini et al., 2001; Müller et al., 2007; Groh et al., 2012). In a recent approach targeting the CSF-1/CSF-1R axis pharmacologically, the same genetically caused neuropathies were substantially alleviated (Klein et al., 2015), further supporting the above-mentioned proof-of-principle studies and additionally displaying a potential translational aspect. In the present study, we further confirmed the pathogenetic significance of CSF-1 by a reciprocal approach, namely by homozygous (over)expression of all CSF-1 isoforms in Cx32def/Csflop/TgC2 mice resulting in an increased number of pathogenetic macrophages and strongly exacerbated neuropathological phenotype.

The major aim of the present study, however, was to investigate the individual roles of the secreted spCSF-1 versus the membrane-spanning csCSF-1 isoform. We showed that $C \times 32$ deficiency increased the expression of Csf1 mRNA in mice hemizygously expressing the different transgenes to levels similar to those observed in Cx32def/Csflwt mice and that twice these levels were exhibited in mice homozygously expressing the transgenes. Of note, mice obtained with either the CS5 or SPP2 on the Csf1op background are devoid of all other isoforms (Dai et al., 2004; Nandi et al., 2006), an important prerequisite in unequivocally deciphering the functions of each isoform. Based on our previous observations that CSF-1 transcripts and protein were predominantly expressed by endoneurial fibroblasts (Groh et al., 2012) and that these cells were often associated with endoneurial macrophages via close membrane-to-membrane associations in normal as well as in diseased nerves (Kroner et al., 2005; Groh et al., 2012), we initially expected that csCSF-1 might regulate the proliferation and pathogenic activation of macrophages in our disease model. According to the present data, both spCSF-1 and csCSF-1 elevate the number of endoneurial macrophages to a similar degree. However, we showed that whereas spCSF-1 mediates macrophage activation and fosters disease progression in Cx32def mice, csCSF-1 inhibits expression of activation markers and dampens neuropathy, even when overexpressed. Furthermore, our quantitative electron microscopic data revealed that spCSF-1 is prominently involved in the generation of axonopathic features, abnormal myelin profiles and the appearance of foamy (phagocytosing) macrophages, whereas regenerative features are more related to csCSF-1. Another interesting observation was that both isoforms appear to be expressed by distinct cell types: whereas the disease-promoting spCSF-1 was expressed by the endoneurial fibroblasts, csCSF-1 was found mainly expressed by macrophages, meaning that the membrane-related isoform may regulate macrophage activity by an autocrine mechanism. Alternatively, it is possible that juxtacrine/paracrine mechanisms occur between neighboring macrophages, a particularly interesting concept as we noticed that pathogenic macrophages usually occur in clusters when executing their detrimental function (Groh et al., 2015b). This kind of regulation between or within macrophages could be a self-limiting mechanism to avoid exacerbation and/or to terminate their detrimental functions. The identification of two distinct sources expressing different CSF-1 isoforms supports and extends our previous findings showing that both endoneurial fibroblasts and macrophages can express CSF-1, with fibroblasts being the major source in myelin mutant nerves (Groh et al., 2012). At present, we cannot fully exclude that our approach of quantifying total CSF-1 in the different trans- 
genic mice might be confounded by effects of the specific isoform on its own expression or other effects on CSF-1-expressing cells. However, the finding that macrophage-derived CSF-1 is much less pathogenic in myelin mutant nerves may explain our previous observation that nerve-immigrating macrophages derived from bone marrow of wt mice (and hence likely expressing some csCSF-1) do not restore or aggravate neuropathy in P0het/Csf1op double-mutants (Müller et al., 2007). Moreover, it is unlikely that spCSF-1 directly acts on the cellular source of this isoform as endoneurial fibroblasts are devoid of the CSF-1 receptor (Carenini et al., 2001).

Interestingly, the spCSF-1-expressing fibroblasts are preferentially associated with macrophages. Although one would not expect that an intimate cell-cell contact between donor and recipient cells is obligatory for function when a ligand is secreted, the tight association between fibroblasts and macrophages will avoid long diffusion pathways of the ligand and ensure a reliable cell-cell communication via the secreted ligand and juxtaposed trans-located receptors. Furthermore, in the case of spCSF-1, a chondroitin sulfate-containing proteoglycan, it is possible that significant amounts are retained in the glycocalyx of the secreting fibroblast. However, it is also possible that the tight cell contacts are necessary for other communication events between the cell partners implicating membrane-bound signaling molecules, like RANK-RANK-L, an important regulator system of genesis and function of osteoclasts and disease (Teitelbaum, 2000; Trouvin and Goeb, 2010) and also expressed by apposed macrophages-fibroblasts in the mutant nerves (Groh et al., 2012). The underlying mechanism leading to the tight apposition of spCSF-1-expressing fibroblasts and macrophages awaits further research. Similarly, the detailed regulation of fibroblast numbers remains presently enigmatic, although our study suggests that both Cx32 deficiency and the csCSF-1 isoform might be directly or indirectly implicated. Thus, it is plausible to assume that Schwann cell-derived signals (in response to loss of $\mathrm{Cx} 32$ ) partially contribute to the fibroblast increase in the mutants. In addition, csCSF-1, expressed by macrophages, might act in an autocrine fashion to bind and activate the CSF-1R to induce the expression of fibroblast growth factors, such as platelet-derived growth factors (Glim et al., 2013).

It is reported that all CSF-1 isoforms are dimeric, share the $150 \mathrm{~N}$-terminal amino acids required for biological activities and have distinct, but broadly overlapping functions (Dai et al., 2004; Nandi et al., 2006; Stanley and Chitu, 2014). Moreover, there is only one known CSF-1 receptor, a tyrosine kinase, encoded by the c-fms protooncogene, with a unique binding domain for all CSF-1 isoforms and also for the alternative ligand, IL-34 (Douglass et al., 2008; Stanley and Chitu, 2014). Thus, the mechanistic question emerges as to how the spCSF-1 and csCSF-1 isoforms, binding to identical receptor domains, can fulfill distinct, even antagonistic, functions with regard to detrimental macrophage activation and pathology. Signaling in cells coexpressing receptor tyrosine kinases and cognate membrane-spanning receptors has been proven difficult to study and no studied paradigms exist. However, the locations of the activation and the trafficking of CSF-1 receptors in macrophages expressing membrane-spanning CSF-1 are likely to be quite different from those of the well studied CSF-1 receptor activation and trafficking induced by soluble CSF-1 and major differences in downstream signaling are likely to result.

Our observation that the spCSF-1 isoform fosters pathogenic features in models for inherited peripheral neuropathies may have important pathomechanistic implications. Although we previously considered the myelin-phagocytosing function of macrophages as most detrimental for nerve integrity (Carenini et al., 2001; Groh et al., 2012), we recently modified and extended our view. By using $C \times 32 h e t\left(C \times 32^{+/-}\right)$mice displaying both Cx32-positive and -negative Schwann cells in one and the same nerve, we showed that pathogenic macrophage clusters not only precisely associate with mutant as opposed to Cx32-positive Schwann cells, but lead to Schwann cell dedifferentiation that likely causes or amplifies axonal damage. Most importantly, this dedifferentiation phenotype and ongoing neuropathy could be completely blocked by the absence of CSF-1 (Groh et al., 2015b). Based on our present observations, it is tempting to speculate that the dedifferentiating function of macrophages is predominantly mediated by spCSF-1. Further studies are necessary to identify the macrophage-borne molecular players that lead to Schwann cell dedifferentiation and potentially axonopathy, and whether these players are indeed upregulated by spCSF-1 and possibly dampened by csCSF-1.

Our study identifying partially overlapping (macrophage numbers) but also antagonistic functions (in macrophage activation and pathogenesis) of the spCSF-1 and csCSF-1 isoforms may have substantial translational implications for CMT1 neuropathies. We have recently shown that in models for two distinct forms of CMT1, Cx32def mice, and P0het mice, representing CMT1X and $1 \mathrm{~B}$, respectively, systemic long-term treatment with a CSF-1 receptor inhibitor (CSF$1 \mathrm{Ri})$ led to substantially ameliorated neuropathy, in the absence of unwanted side effects (Klein et al., 2015). However, there were differences in disease amelioration induced by CSF-1Ri and the amelioration observed in myelin/Csf1op double-mutants (Klein et al., 2015). As opposed to the latter, upon CSF-1Ri treatment, there was robust macrophage ablation below wt levels, similar to effects on microglia in the CNS when a similar inhibitor was used (Elmore et al., 2014; Rice et al., 2015). This robust decline of nerve macrophages (and microglia in the CNS) is likely caused by inhibition of macrophage survival mediated by the alternative ligand IL-34 (Wei et al., 2010; Wang and Colonna, 2014). Although lack or reduced numbers of macrophages and microglial cells are compatible with long-term survival of laboratory animals (Elmore et al., 2014; Klein et al., 2015; Rice et al., 2015), macrophage depletion could be considered as a potential risk factor for long-term treatment of humans, especially children, suffering from a nonfatal disease, as the requirement for CSF-1 and the CSF-1 receptor for survival is primarily developmental (Dai et al., 2002). Therefore, it may be a reasonable alternative to block the spCSF-1 isoform with corresponding antibodies and to leave the CSF-1 receptor intact for IL-34related macrophage survival. Of note, long-term treatment approaches with blocking antibodies are generally an approved and advancing option for treatment of neuroinflammatory disorders (Deiß et al., 2013). An alternative option could be to identify low-molecular-weight mimetics of the disease-mitigating csCSF-1 isoform to physiologically counterbalance the detrimental spCSF-1.

\section{References}

Anzini P, Neuberg DH, Schachner M, Nelles E, Willecke K, Zielasek J, Toyka KV, Suter U, Martini R (1997) Structural abnormalities and deficient maintenance of peripheral nerve myelin in mice lacking the gap junction protein connexin 32. J Neurosci 17:4545-4551. Medline 
Carenini S, Mäurer M, Werner A, Blazyca H, Toyka KV, Schmid CD, Raivich G, Martini R (2001) The role of macrophages in demyelinating peripheral nervous system of mice heterozygously deficient in p0. J Cell Biol 152:301-308. CrossRef Medline

Cecchini MG, Dominguez MG, Mocci S, Wetterwald A, Felix R, Fleisch H, Chisholm O, Hofstetter W, Pollard JW, Stanley ER (1994) Role of colony stimulating factor- 1 in the establishment and regulation of tissue macrophages during postnatal development of the mouse. Development 120:1357-1372. Medline

Dai XM, Ryan GR, Hapel AJ, Dominguez MG, Russell RG, Kapp S, Sylvestre V, Stanley ER (2002) Targeted disruption of the mouse colonystimulating factor 1 receptor gene results in osteopetrosis, mononuclear phagocyte deficiency, increased primitive progenitor cell frequencies, and reproductive defects. Blood 99:111-120. CrossRef Medline

Dai XM, Zong XH, Sylvestre V, Stanley ER (2004) Incomplete restoration of colony-stimulating factor 1 (CSF-1) function in CSF-1-deficient Csflop/ Csflop mice by transgenic expression of cell surface CSF-1. Blood 103: 1114-1123. CrossRef Medline

Deiß A, Brecht I, Haarmann A, Buttmann M (2013) Treating multiple sclerosis with monoclonal antibodies: a 2013 update. Expert Rev Neurother 13:313-335. CrossRef Medline

Douglass TG, Driggers L, Zhang JG, Hoa N, Delgado C, Williams CC, Dan Q, Sanchez R, Jeffes EW, Wepsic HT, Myers MP, Koths K, Jadus MR (2008) Macrophage colony stimulating factor: not just for macrophages anymore! A gateway into complex biologies. Int Immunopharmacol 8: 1354-1376. CrossRef Medline

Elmore MR, Najafi AR, Koike MA, Dagher NN, Spangenberg EE, Rice RA, Kitazawa M, Matusow B, Nguyen H, West BL, Green KN (2014) Colony-stimulating factor 1 receptor signaling is necessary for microglia viability, unmasking a microglia progenitor cell in the adult brain. Neuron 82:380-397. CrossRef Medline

Fledrich R, Stassart RM, Sereda MW (2012) Murine therapeutic models for Charcot-Marie-Tooth (CMT) disease. Br Med Bull 102:89-113. CrossRef Medline

Glim JE, Niessen FB, Everts V, van Egmond M, Beelen RH (2013) Platelet derived growth factor-CC secreted by M2 macrophages induces alphasmooth muscle actin expression by dermal and gingival fibroblasts. Immunobiology 218:924-929. CrossRef Medline

Groh J, Weis J, Zieger H, Stanley ER, Heuer H, Martini R (2012) Colonystimulating factor-1 mediates macrophage-related neural damage in a model for Charcot-Marie-Tooth disease type 1X. Brain 135:88-104. CrossRef Medline

Groh J, Klein D, Kroner A, Martini R (2015a) Inflammation in the pathogenesis of inherited peripheral neuropathies. In: Neuroinflammation: new insights into beneficial and detrimental functions, Ed 1 (David S, ed), pp 123-137. Hoboken, New Jersey: Wiley.

Groh J, Klein I, Hollmann C, Wettmarshausen J, Klein D, Martini R (2015b) CSF-1-activated macrophages are target-directed and essential mediators of Schwann cell dedifferentiation and dysfunction in Cx32-deficient mice. Glia 63:977-986. CrossRef Medline

Hiroyasu S, Chinnasamy P, Hou R, Hotchkiss K, Casimiro I, Dai XM, Stanley ER, Sibinga NE (2013) Donor and recipient cell surface colony stimulating factor-1 promote neointimal formation in transplant-associated arteriosclerosis. Arterioscler Thromb Vasc Biol 33:87-95. CrossRef Medline

Huynh D, Dai XM, Nandi S, Lightowler S, Trivett M, Chan CK, Bertoncello I, Ramsay RG, Stanley ER (2009) Colony stimulating factor-1 dependence of paneth cell development in the mouse small intestine. Gastroenterology 137:136-144.e3. CrossRef Medline

Jerath NU, Shy ME (2015) Hereditary motor and sensory neuropathies: understanding molecular pathogenesis could lead to future treatment strategies. Biochim Biophys Acta 1852:667-678. CrossRef Medline

Johnson NE, Heatwole CR, Dilek N, Sowden J, Kirk CA, Shereff D, Shy ME, Herrmann DN (2014) Quality-of-life in Charcot-Marie-Tooth disease: the patient's perspective. Neuromuscul Disord 24:1018-1023. CrossRef Medline

Klein D, Patzkó Á, Schreiber D, van Hauwermeiren A, Baier M, Groh J, West BL, Martini R (2015) Targeting the colony-stimulating factor-1 receptor alleviates two forms of Charcot-Marie-Tooth disease in mice. Brain 138:3193-3205. CrossRef Medline
Kobsar I, Berghoff M, Samsam M, Wessig C, Mäurer M, Toyka KV, Martini R (2003) Preserved myelin integrity and reduced axonopathy in connexin32-deficient mice lacking the recombination activating gene-1. Brain 126:804-813. CrossRef Medline

Kroner A, Mäurer M, Kohl B, Kobsar I, Martini R (2005) Endoneurial fibroblast-like cells express the hematopoietic stem cell marker CD34 and form cell contacts with resident and phagocytosing macrophages in normal mice and demyelinating mutants. J Peripher Nerv Syst 10:46.

Müller M, Berghoff M, Kobsar I, Kiefer R, Martini R (2007) Macrophage colony stimulating factor is a crucial factor for the intrinsic macrophage response in mice heterozygously deficient for the myelin protein P0. Exp Neurol 203:55-62. CrossRef Medline

Nandi S, Akhter MP, Seifert MF, Dai XM, Stanley ER (2006) Developmental and functional significance of the CSF-1 proteoglycan chondroitin sulfate chain. Blood 107:786-795. CrossRef Medline

Nelles E, Bützler C, Jung D, Temme A, Gabriel HD, Dahl U, Traub O, Stümpel F, Jungermann K, Zielasek J, Toyka KV, Dermietzel R, Willecke K (1996) Defective propagation of signals generated by sympathetic nerve stimulation in the liver of connexin32-deficient mice. Proc Natl Acad Sci U S A 93:9565-9570. CrossRef Medline

Pareyson D, Saveri P, Piscosquito G (2014) Charcot-Marie-Tooth disease and related hereditary neuropathies: from gene function to associated phenotypes. Curr Mol Med 14:1003-1033. Medline

Patzko A, Shy ME (2011) Update on Charcot-Marie-Tooth disease. Curr Neurol Neurosci Rep 11:78-88. CrossRef Medline

Pixley FJ, Stanley ER (2004) CSF-1 regulation of the wandering macrophage: complexity in action. Trends Cell Biol 14:628-638. CrossRef Medline

Price LK (1992) Biosynthetic studies of membrane associated and secreted L cell CSF-1. In: Albert Einstein College of Medicine. New York: Yeshiva University.

Price LK, Choi HU, Rosenberg L, Stanley ER (1992) The predominant form of secreted colony stimulating factor-1 is a proteoglycan. J Biol Chem 267:2190-2199. Medline

Rice RA, Spangenberg EE, Yamate-Morgan H, Lee RJ, Arora RP, Hernandez MX, Tenner AJ, West BL, Green KN (2015) Elimination of microglia improves functional outcomes following extensive neuronal loss in the hippocampus. J Neurosci 35:9977-9989. CrossRef Medline

Richard L, Topilko P, Magy L, Decouvelaere AV, Charnay P, Funalot B, Vallat JM (2012) Endoneurial fibroblast-like cells. J Neuropathol Exp Neurol 71:938-947. CrossRef Medline

Ryan GR, Dai XM, Dominguez MG, Tong W, Chuan F, Chisholm O, Russell RG, Pollard JW, Stanley ER (2001) Rescue of the colony-stimulating factor 1 (CSF-1)-nullizygous mouse (Csf1(op)/Csf1(op)) phenotype with a CSF-1 transgene and identification of sites of local CSF-1 synthesis. Blood 98:74-84. CrossRef Medline

Stanley ER, Chitu V (2014) CSF-1 receptor signaling in myeloid cells. Cold Spring Harb Perspect Biol 6:a021857. CrossRef Medline

Teitelbaum SL (2000) Bone resorption by osteoclasts. Science 289: 1504-1508. CrossRef Medline

Trouvin AP, Goëb V (2010) Receptor activator of nuclear factor-kappaB ligand and osteoprotegerin: maintaining the balance to prevent bone loss. Clin Interv Aging 5:345-354. CrossRef Medline

Wang Y, Colonna M (2014) Interkeukin-34, a cytokine crucial for the differentiation and maintenance of tissue resident macrophages and Langerhans cells. Eur J Immunol 44:1575-1581. CrossRef Medline

Wei S, Nandi S, Chitu V, Yeung YG, Yu W, Huang M, Williams LT, Lin H, Stanley ER (2010) Functional overlap but differential expression of CSF-1 and IL-34 in their CSF-1 receptor-mediated regulation of myeloid cells. J Leuko Biol 88:495-505. CrossRef Medline

Wiktor-Jedrzejczak W, Bartocci A, Ferrante AW Jr, Ahmed-Ansari A, Sell KW, Pollard JW, Stanley ER (1990) Total absence of colonystimulating factor 1 in the macrophage-deficient osteopetrotic (op/ op) mouse. Proc Natl Acad Sci U S A 87:4828-4832. CrossRef Medline

Yoshida H, Hayashi SI, Kunisada T, Ogawa M, Nishikawa S, Okamura H, Sudo T, Shultz LD, Nishikawa S (1990) The murine mutation osteopetrosis is in the coding region of the macrophage colony stimulating factor gene. Nature 345:442-444. CrossRef Medline 